\title{
THE DIRAC EQUATION IN GEOMETRIC QUANTIZATION
}

\author{
ANDREJ BÓNA
}

\begin{abstract}
The coadjoint orbit of the restricted Poincaré group corresponding to a mass $\mathrm{m}$ and spin $1 / 2$ is described. The orbit is quantized using the geometric quantization. To include the discrete symmetries, one has to induce the irreducible representation of the restricted Poincaré group obtained by the quantization procedure to the full Poincaré group. The new representation is reducible and the reduction to an irreducible representation corresponds to the Dirac equation.
\end{abstract}

\section{Contents}

1. Introduction

1.1. Group theory

1.2. Geometric quantization 2

1.3. Summary of the results 3

1.4. The organization of the paper 5

2. Short introduction to geometric quantization 5

3. The Poincaré group and algebra 6

3.1. Action of the Lorentz group on spinors 10

3.2. Tangent vectors 11

3.3. Discrete symmetries 11

3.4. The Orbit 13

3.5. Coordinates 13

3.6. Symplectic structure 14

3.7. Hamiltonian vector fields 14

4. Quantization 15

4.1. Prequantization $\quad 15$

4.2. Polarization 17

References 24

The author would like to thank Jẹdrzej Śniatycki for his help and support. 


\section{INTRODUCTION}

The Dirac equation is one of the most important equations in relativistic quantum physics as it describes particles of spin $1 / 2$, and both electron and protons have spin 1/2. When P.A.M.Dirac introduced this equation [5] in 1928, while his reasoning was original and insightful, his derivation of the equation did not shed much light on the meaning of the equation. The purpose of this paper is to interpret the Dirac equation from geometric-quantization point of view and thus provide more understanding of the topic.

1.1. Group theory. In quantum mechanics, the states of a system are represented by vectors (or rays) of a Hilbert space and observables correspond to self-adjoint operators. In the case of a free (isolated) relativistic system, one requires the theory to be invariant under the Poincaré group. More precisely, there is one-to-one correspondence between states corresponding to any two different observers observing the same system and the transition probabilities for the two observers are equal. These conditions imply an existence of a unitary (or antiunitary) representation of the Poincaré group in the Hilbert space.

If one assumes from the very beginning the invariance of the theory under the Poincaré group (or some other group), one can look at possible unitary representations of the group and decide if a given representation is physically meaningful or not. Moreover, if one is interested in description of elementary quantum systems, one restricts the representations to only the irreducible ones [8]. This way, using only the representation theory, one can obtain physically meaningful results. Perhaps the most important one stems from the time translations given by the group representation. This one parameter subgroup of unitary operators corresponds to the time evolution of the system, or, in other words, represents the equation of motion for the free system.

It is possible to use the pure group theory to find the Dirac equation or other relativistic wave equations. This has been done, for example, in [3].

The knowledge of the representations of the group, however, does not constitute a full physical theory. A model based solely on a representation of the group does not contain any information on the observable quantities of the system. To obtain this information, one needs to consult the corresponding classical system. To relate the classical system with a quantum one, one can quantize the corresponding classical system. It was noted by Souriau [13] and Kostant [7], that coadjoint orbits have a symplectic structure and thus represent a classical mechanical system. The underlying classical system in the case of a free relativistic spinning particle is a coadjoint orbit of the Poincaré group. To quantize this system, the method of geometric quantization is used.

1.2. Geometric quantization. The canonical quantization is perhaps the most known method of relating classical and quantum physics. It associates to a classical observable $f(x, p)$ an operator $f\left(x,-i \hbar \frac{\partial}{\partial x}\right)$ on the Hilbert space of square integrable functions $\psi(x)$. This method has many drawbacks, among which the 
most obvious one is the problem of ordering of $x$ s and $q \mathrm{~s}$. The geometric quantization generalizes the canonical quantization to a coordinate free theory and all the structures have a nice geometrical meaning. Historically, the geometric quantization started by publications of Souriau [13] and Kostant [7] and later that of Blattner [4]. A prequantization of the Poincaré group was done already in 1969 [10], but in this paper the construction of the prequantization is done in a different way.

1.3. Summary of the results. One can represent the trivial line bundle over the coadjoint orbit $\mathcal{O}$ of the Poincaré group, corresponding to mass $m$ and spin $s$, by the space of sections $\lambda$ of a trivial line bundle over the space

$$
M=\left\{(x, p, z) \in \mathbb{R}^{1,3} \times \mathbb{R}^{1,3} \times \mathbb{C}^{2}\right\}
$$

which are restricted by the conditions

$$
\begin{aligned}
\nabla_{X_{p^{2}}} \lambda & =0 \\
\nabla_{X_{z p \bar{z}}} \lambda & =0
\end{aligned}
$$

and

$$
\begin{aligned}
P_{p^{2}-m^{2}} \lambda & =0 \\
P_{z p \bar{z}-s / 2 \pi} \lambda & =0,
\end{aligned}
$$

where $P_{f}$ is a prequantum operator corresponding to function $f$. Here the vector fields $X_{p^{2}}$ and $X_{z p \bar{z}}$ are the Hamiltonian vector fields, corresponding to functions $p^{2}$ and $z p \bar{z}$, given by the symplectic form $\omega$, which, restricted to the orbit $\mathcal{O}$, is the Kostant-Soriau form. The operators $P$ are the prequantized operators in the trivial bundle over $M$.

The quantization in this representation is given by introducing the polarization spanned by the vectors $\partial_{x}$ and $\bar{w}^{A} \partial_{\bar{z}^{A}}$. The quantized space is thus represented by sections of the trivial line bundle over $M$ with restrictions

$$
\begin{aligned}
\nabla_{\partial_{x}} \lambda & =0 \\
\nabla_{\bar{w}^{A} \partial_{\bar{z} A}} \lambda & =0
\end{aligned}
$$

and

$$
\begin{aligned}
\nabla_{X_{z p \bar{z}}} \lambda & =0 \\
P_{p^{2}-m^{2}} \lambda & =0 \\
P_{z p \bar{z}-s / 2 \pi} \lambda & =0 .
\end{aligned}
$$

Here, formally, the prequantized operators are the same as quantized operators, since the flows of the Hamiltonian vector fields $X_{p^{2}-m^{2}}$ and $X_{z p \bar{z}-s / 2 \pi}$ preserve the polarization spanned by the vectors $\partial_{x}$ and $\bar{w}^{A} \partial_{\bar{z}^{A}}$. Note also that the condition on the sections from the prequantization

$$
\nabla_{X_{p^{2}}} \lambda=0
$$


has vanished, since this condition is now included in the condition of covariant constancy along the polarization. This is implied by the fact that the vector field $X_{p^{2}}=-2 p \partial_{x}$ belongs to the polarization. This way the space of quantized sections $H_{p}$ is of the following form

$$
H_{p}=\left\{\begin{array}{c}
\lambda \mid \nabla_{\partial_{x}} \lambda=0, \nabla_{\bar{w}^{A} \partial_{\bar{z} A}} \lambda=0, \nabla_{X_{z p \bar{z}}} \lambda=0 \\
P_{p^{2}-m^{2}} \lambda=0, P_{z p \bar{z}-s / 2 \pi} \lambda=0
\end{array}\right\} .
$$

The action of the one parameter subgroup of the restricted Poincaré group, generated by an element $\xi=(A, a)$ of the Lie algebra, on the elements of $H_{p}$ is given by operators $U_{\xi}^{t}$ acting as

$$
U_{\xi}^{t} \lambda=\left(\psi \circ \phi_{t}\right) \lambda_{0}
$$

where $\lambda=\psi \lambda_{0}$ and $\phi_{t}$ is the flow of the vector field generated by $\xi=(A, a)$.

The action of the discrete symmetries is not, however, well defined on this space. The discrete symmetries act on the spinorial part as antilinear operators, and thus they don't preserve the polarization. To define the action of the discrete symmetries, one can induce the representation of the Poincaré group. To do this, one can take the representation of the restricted Poincaré group which lives in the space $H_{p}$. One then takes the image of $H_{p}$ under the discrete symmetries in $H$, space of sections of the line bundle over $M$. The product of these four spaces forms the new representation space, in which the discrete symmetries act as they act in the prequantized bundle:

$$
\begin{aligned}
& T \psi=\psi \circ T \\
& P \psi=\psi \circ P .
\end{aligned}
$$

One can further combine the elements of the four spaces into one vector valued function. This way one can write

$$
\psi=e^{2 \pi i\langle p, x\rangle}\left(\begin{array}{c}
z^{A} \xi_{A}(p) \\
\bar{z}_{A^{\prime}} \eta^{A^{\prime}}(p)
\end{array}\right)
$$

and

$$
\begin{aligned}
U_{(A, a)}^{t}\left[e^{2 \pi i\langle p, x\rangle}\left(\begin{array}{c}
\xi_{A} \\
\eta^{A^{\prime}}
\end{array}\right)(p)\right] & =e^{2 \pi i\langle p, a\rangle}\left(\begin{array}{c}
e^{A^{T} t} \xi_{A} \\
e^{-\bar{A} t} \eta^{A^{\prime}}
\end{array}\right)\left(e^{A t} p\right) \\
T \psi(x, p, z) & =e^{2 \pi i\langle p, x\rangle}\left(\begin{array}{c}
\bar{z}_{A^{\prime}} \eta^{A^{\prime}}(T p) \\
z^{A} \xi_{A}(T p)
\end{array}\right) \\
P \psi(x, p, z) & =e^{2 \pi i\langle p, x\rangle}\left(\begin{array}{c}
\bar{z}_{A^{\prime}} \eta^{A^{\prime}}(P p) \\
z^{A} \xi_{A}(P p)
\end{array}\right)
\end{aligned}
$$

This representation is not irreducible; there is the operator $D$ that commutes with all elements of the representation of the Poincaré group. The operator $D$ acts on the sections represented as $\lambda=\psi \lambda_{0}$ as follows:

$$
D \psi(x, p, z)=D\left[e^{2 \pi i\langle p, x\rangle}\left(\begin{array}{c}
z^{A} \xi_{A}(p) \\
\bar{z}_{A^{\prime}} \eta^{A^{\prime}}(p)
\end{array}\right)\right]=e^{2 \pi i\langle p, x\rangle}\left(\begin{array}{c}
z^{A} p_{A A^{\prime}} \eta^{A^{\prime}}(p) \\
\xi_{A}(p) p^{A A^{\prime}} \bar{z}_{A^{\prime}}
\end{array}\right) .
$$


To get an irreducible representation, one has to investigate the eigenspaces of the operator $D$. The operator $D$ has eigenvalues $\pm m$. Thus, the wave functions of the irreducible representation must satisfy the equation

$$
D \psi= \pm m \psi \text {. }
$$

This is the Dirac equation.

1.4. The organization of the paper. This paper is divided into two main parts. In the first part, the Poincaré group is discussed. This part is mainly a technical elaboration of the construction of the coadjoint orbits and the related symplectic structure. The construction of the orbit is done with help of two-spinors. This construction has been motivated by [14].

The second part is devoted to the geometric quantization of the Poincaré group. It is in this part of the paper that the most of the new and interesting work is presented.

At the beginning of the paper, a short summary of the geometric quantization is presented.

\section{Short introduction to GEOMETRIC QuANTIZATION}

Let $M$ be a $2 n$ dimensional symplectic manifold with a symplectic structure given by a 2 -form $\omega$. One considers a complex line bundle $(L, \pi, M)$ over the manifold $M$ together with a connection $\alpha \in \Lambda(L)$ such that $\mathrm{d} \alpha=\pi^{*} \omega$. The existence of such a line bundle is guaranteed provided that so called integrality condition is satisfied:

$$
\int \omega \in \mathbb{Z}
$$

where the integration is over each closed oriented two-surface in $M$.

One can construct a Hilbert space $H$ of square integrable sections $\lambda$ of $L$, where the scalar product is given by

$$
\left(\lambda_{1}, \lambda_{2}\right)=\int_{M}\left(\lambda_{1}(m), \lambda_{2}(m)\right)_{m} \omega^{n} .
$$

Here the symbol $(., .)_{m}$ represents the product on the fibre $\pi^{-1}(m) \cong \mathbb{C}$.

For each differentiable function $f$ on $M$, there is a Hamiltonian vector field $X_{f}$, such that $\left.X_{f}\right\lrcorner \omega=-\mathrm{d} f$. It is possible to lift the Lie algebra of the Hamiltonian vector fields $\left[X_{f}, X_{g}\right]=X_{\{f, g\}}$, here $\{.,$.$\} is the Poisson bracket, to the line bundle$ $L$ to obtain a faithful representation of the Poisson algebra $\{f, g\}^{1}$. To do that, one defines to each $f$ a self-adjoint operator $P_{f}$ acting on $H$ as

$$
P_{f}=\nabla_{X_{f}}-2 \pi i f
$$

where $\nabla$ is the covariant derivative given by the connection $\alpha$. The representation $P_{f}$ of the Poincare algebra is called prequantization. The Hilbert space $H$ has

\footnotetext{
${ }^{1}$ The hamiltonian vector fields are not a faithful representation of the Poisson algebra because all constant functions map to the zero vectorfield.
} 
still too big to satisfy the Heisenberg principle. To deal with this difficulty, one introduces a polarization on the manifold $M$ and restricts the space of sections to sections that are covariantly constant along this polarization. This, however, destroys the Hilbert space, since these sections are not square integrable with respect to the previously introduced scalar product. One can still introduce a scalar product with respect to which the covariantly constant sections along the polarization can be square integrable. This is done with help of half-forms. The topic is too extensive to be covered in a brief overview, hence the reader is referred to literature [4]. The object similar to a half-form is a half-density, which is much easier to define and suffices for the purpose of this overview.

A $d$-density is an element of the associated fibre bundle to the frame bundle $F N$ of $N$ with the fibre $\mathbb{R}$ and action of $G l(n, \mathbb{R})$ on the fibre being

$$
g x=|\operatorname{det} g|^{d} x,
$$

where $x \in \mathbb{R}$ and $g \in G l(n, \mathbb{R})$. A $d$-density $\mu$ can be represented by a complex valued function $\mu^{\#}$ on the frame bundle $F N$, such that for $g \in G l(n, \mathbb{R})$ and each $w \in F N$,

$$
\mu^{\#}(w g)=|\operatorname{det} g|^{d} \mu(w) .
$$

The pair of two half-densities $\mu_{1}$ and $\mu_{2}$ can define a one-density $\left(\mu_{1}, \mu_{2}\right)$ as

$$
\left(\mu_{1}, \mu_{2}\right)^{\#}(w)=\overline{\mu_{1}^{\#}(w)} \mu_{2}^{\#}(w) \text {. }
$$

A one-density can be integrated over $N$.

One can define for a polarization $F$ two other distributions, namely, $D=$ $F \cap \bar{F} \cap T M$ and $E=(F+\bar{F}) \cap T M$. For so called strongly admissible polarization $F, D$ and $E$ are involutive distributions, the spaces $M / D$ and $M / E$ are manifolds and the projections $\pi_{E}: M \rightarrow M / E, \pi_{D}: M \rightarrow M / D$ and $\pi_{E D}: M / D \rightarrow M / E$ are submersions. If $F$ is strongly admissible, then for each integral manifold $P$ of $E, Q=\pi_{E D}^{-1}(P)$ is a symplectic manifold with a polarization $F_{Q}$ such that $F_{Q} \cap \bar{F}_{Q}=0$ and $F_{Q}=\left.T \pi_{D} F\right|_{P}$ with the symplectic form $\omega_{Q}$ such that $\left.\omega\right|_{P}=$ $\pi_{D}^{*} \omega_{Q}$. This allows to define a scalar product for covariantly constant sections along the polarization $F$ in an extended space with elements $\lambda \otimes \mu$

$$
\left(\lambda_{1} \otimes \mu_{1}, \lambda_{2} \otimes \mu_{2}\right)=\int_{M / E}\left(\int_{Q} \pi_{D}^{*}\left(\lambda_{1}, \lambda_{2}\right) \omega_{Q}^{n-d}\right)\left(\mu_{1}, \mu_{2}\right),
$$

where $\mu_{i}$ are densities on $M / E$ and $d$ is dimension of $D$. This scalar product can be used to define the Hilbert space $\mathcal{H}$.

It is possible to define quantized operators on $\mathcal{H}$ corresponding to a function whose Hamiltonian vector field preserves the polarization. The details can be found in $[12]$.

\section{The Poincaré group And Algebra}

The Poincaré group is a group of linear isomorphisms of the Minkowski space that preserve the distance. More precisely, one can express the elements of the Poincaré group as follows. 
An element $g$ of the Poincaré group $G$ can be represented by a pair

$$
g=(\Lambda, l),
$$

where $\Lambda$ is a matrix and $l$ is a vector from the Minkowski space. The action $\rho$ of the group on the vector $x$ from the Minkowski space is

$$
(\rho(g) x)^{\alpha}=\Lambda_{\beta}^{\alpha} x^{\beta}+l^{\alpha} .
$$

The Einstein summation convention is used here, as well as in the rest of this document. The multiplication and the inverse element are given for $g=(\Lambda, l)$ and $h=(\Delta, d)$ by

$$
g h=(\Lambda \Delta, \Lambda d+l), g^{-1}=\left(\Lambda^{-1},-\Lambda^{-1} l\right) .
$$

Since the scalar product on the Minkowski space $\mathbb{R}^{1,3}$ is given by the tensor $g_{\alpha \beta}$, that has signature,,,+--- , and $\Lambda$ preserves the scalar product, the matrix $\Lambda$ must satisfy the following equation: $\Lambda_{\beta}^{\alpha} \Lambda_{\gamma}{ }^{\beta}=g_{\gamma}^{\alpha}$. In this expression, the lowering of the indices with help of $g_{\alpha \beta}$ is used, e.g. $x_{\alpha}=g_{\alpha \beta} x^{\beta}$.

An element $\xi$ of the Poincaré algebra $\mathfrak{g}$ can be represented by the pair $\xi=$ $\left(A_{\beta}^{\alpha}, a^{\alpha}\right)$, where the tensor $A^{\alpha \gamma}=A_{\beta}^{\alpha} g^{\beta \gamma}$ must be antisymmetric, $A^{\alpha \gamma}=-A^{\gamma \alpha}$, and $a$ is a vector from the Minkowski space $\mathbb{R}^{1,3}$. In this case, the exponential map exp $: \mathfrak{g} \longrightarrow G$ is given by $\exp t \xi=\left(e^{t A}, t a\right)$. Here, the expression $e^{t A}$ is to be understood as the standard exponentiation of the matrix $A_{\beta}^{\alpha}$.

An element $\alpha$ of the dual space $\mathfrak{g}^{*}$ to the space $\mathfrak{g}$ can be expressed as the pair $\alpha=\left(M_{\alpha}^{\beta}, p_{\alpha}\right)$ that acts on the element $\xi=(A, a) \in \mathfrak{g}$ as

$$
\alpha(\xi)=\langle M, A\rangle-\langle p, a\rangle=1 / 2 \operatorname{Tr} M A-p a=-1 / 2 M_{\alpha \beta} A^{\alpha \beta}-p_{\alpha} a^{\alpha},
$$

where $M_{\alpha}^{\beta} g_{\beta \gamma}=M_{\alpha \gamma}=-M_{\gamma \alpha}=-M_{\gamma}^{\beta} g_{\beta \alpha}$ with $p \in \mathbb{R}^{1,3}$. The adjoint action

$$
A d_{(\Lambda, l)}(A, a)=\left.\frac{\mathrm{d}}{\mathrm{d} t}(\Lambda, l) \exp \{t(A, a)\}(\Lambda, l)^{-1}\right|_{t=0}=\left(\Lambda A \Lambda^{-1},-\Lambda A \Lambda^{-1} l+\Lambda a\right),
$$

together with the evaluation $\alpha(\xi)$, gives the coadjoint action:

$$
\begin{aligned}
\left\langle A d_{(\Lambda, l)}^{*}(M, p),(A, a)\right\rangle & =\left\langle(M, p), A d_{(\Lambda, l)^{-1}}(A, a)\right\rangle \\
& =\left\langle(M, p),\left(\Lambda^{-1} A \Lambda, \Lambda^{-1} A l+\Lambda^{-1} a\right)\right\rangle \\
& =\left\langle\Lambda M \Lambda^{-1}, A\right\rangle-\langle\Lambda p, A l\rangle-\langle\Lambda p, a\rangle .
\end{aligned}
$$

If one defines the wedge product of $x$ and $y$ as $(x \wedge y)^{\alpha \beta}=x^{\alpha} y^{\beta}-y^{\alpha} x^{\beta}$, then the following identity holds:

$$
\begin{aligned}
\langle x,(p \wedge q) y\rangle & =x^{\alpha}(p \wedge q)_{\alpha \beta} y^{\beta}=\frac{1}{2}\left(x^{\alpha}(p \wedge q)_{\alpha \beta} y^{\beta}-y^{\beta}(p \wedge q)_{\beta \alpha} x^{\alpha}\right) \\
& =\frac{1}{2}\left((p \wedge q)_{\alpha \beta}(x \wedge y)^{\alpha \beta}\right)=-\langle p \wedge q, x \wedge y\rangle
\end{aligned}
$$


Using this formula, one can write the expression (2) as

$$
\begin{aligned}
\left\langle\Lambda M \Lambda^{-1}, A\right\rangle-\langle\Lambda p, A l\rangle-\langle\Lambda p, a\rangle & =\left\langle\Lambda M \Lambda^{-1}, A\right\rangle+\langle A, \Lambda p \wedge l\rangle-\langle\Lambda p, a\rangle \\
& =\left\langle\Lambda M \Lambda^{-1}+\Lambda p \wedge l, A\right\rangle-\langle\Lambda p, a\rangle \\
& =\left\langle\left(\Lambda M \Lambda^{-1}+\Lambda p \wedge l, \Lambda p\right),(A, a)\right\rangle,
\end{aligned}
$$

where one uses the fact that any antisymmetric tensor, and thus also $A$, can be expressed as a sum of tensors in a form of $p \wedge q$. This way one can conclude that

$$
A d_{(\Lambda, l)}^{*}(M, p)=\left(\Lambda M \Lambda^{-1}+\Lambda p \wedge l, \Lambda p\right) .
$$

To express the elements of the dual space to the Poincaré algebra in more convenient way, one uses a notion of the dual tensor * $A$ to the tensor $A$ given by the expression: ${ }^{*} A_{\alpha \mu}=\frac{1}{2} \varepsilon_{\alpha \mu \nu \lambda} A^{\nu \lambda}$. The symbol $\varepsilon$ denotes the Kronecker totally antisymmetric tensor. Then, one can use the following theorem:

Theorem 1. Any antisymmetric tensor $M^{\alpha \beta}=-M^{\beta \alpha}$ can be written in a form

$$
M=\left(x \wedge p+^{*}(p \wedge u)\right)
$$

for a given $p$, where $x^{\alpha}=\frac{1}{p^{2}}\left(M^{\alpha \beta} p_{\beta}+t p^{\alpha}\right), u_{\alpha}=\frac{1}{p^{2}}\left(\left({ }^{*} M\right)_{\alpha \beta} p^{\beta}+t^{\prime} p_{\alpha}\right)$ for some real $t$ and $t^{\prime}$. Then automatically $\langle M p, p\rangle=\left\langle\left({ }^{*} M\right) p, p\right\rangle=0$.

Proof. The proof can be simply obtained using formulas in [2].

Based on the previous theorem, one can reparametrize the dual of the Poincaré algebra as follows

$$
(M, p) \rightarrow\left(x^{\alpha}(t)=\frac{M^{\alpha \beta} p_{\beta}+t p^{\alpha}}{p^{2}}, p_{\gamma}, u_{\delta}\left(t^{\prime}\right)=\frac{\left({ }^{*} M\right)_{\delta \varepsilon} p^{\varepsilon}+t^{\prime} p_{\delta}}{p^{2}}\right),
$$

for some real $t, t^{\prime}$, where all points on the lines $x(t)$ and $u(t)$ describe one point on the orbit. The coadjoint action in this representation is given by the formula

$$
A d_{(\Lambda, l)}^{*}(x, p, u)=(\Lambda x+l, \Lambda p, \Lambda u)
$$

since, recalling (2),

$$
\begin{aligned}
A d_{(\Lambda, l)}^{*}\left(x \wedge p+^{*}(p \wedge u), p\right) & =\left(\Lambda(x \wedge p) \Lambda^{-1}+\Lambda^{*}(p \wedge u) \Lambda^{-1}-l \wedge \Lambda p, \Lambda p\right) \\
& =\left(\Lambda x \wedge \Lambda p+\Lambda p \wedge l+^{*}(\Lambda p \wedge \Lambda u), \Lambda p\right) \\
& \longleftrightarrow(\Lambda x+l, \Lambda p, \Lambda u)
\end{aligned}
$$

using $\Lambda(x \wedge p) \Lambda^{-1}=\Lambda x \wedge \Lambda p$.

The coadjoint orbit $\mathcal{O}_{\alpha}=\left\{\beta=A d_{\Lambda, l}^{*} \alpha \mid \alpha \in \mathfrak{g}^{*},(\Lambda, l) \in G\right\}$ of Poincaré group $G$ in the dual $\mathfrak{g}^{*}$ of its algebra passing through the point $\alpha=(M, p)$ is then (5) $\mathcal{O}_{(M, p)}=\left\{(\Lambda x+l, \Lambda p, \Lambda u) \mid x=\frac{1}{p^{2}}(M p+t p), u=\frac{1}{p^{2}}\left(\left(^{*} M\right) p+t^{\prime} p\right)\right\} / \sim$, where the equivalence relation $\sim$ is given by $x \sim x+t p, u \sim u+t^{\prime} p$. The coadjoint action preserves the equivalence classes. 
The group action preserves $p^{2}$ as well as $\langle p \wedge u, p \wedge u\rangle$, since $\langle p \wedge u, p \wedge u\rangle=$ $-p^{2} u^{2}+(p u)^{2}=-p^{2}(u+t p)^{2}+(p \cdot(u+t p))^{2}$. Any orbit is completely determined by these two numbers, since one can choose a representant $u$ from the equivalence class $u+t p$ such that $p u=0$ and thus $\langle p \wedge u, p \wedge u\rangle$ with $p^{2}$ determine $u^{2}$. Since in this paper only the orbits with positive $p^{2}$ are treated, the numbers $p^{2}$ and $\langle p \wedge u, p \wedge u\rangle$ are going to be called $m^{2}$ and $(s / \pi)^{2}$ respectively, for $m$ and $s$ real.

One would like to express the Kostant-Souriau form on the coadjoint orbits. The coordinates $(x, p, u)$ are not the most convenient ones. Better coordinates are expressed with help of two-spinors. Recall that the space $\mathcal{S} \simeq \mathbb{C}^{2}$ of spinors $z^{A}$ is endowed with a symplectic form given by tensor

$$
\epsilon_{A B}=\left(\begin{array}{cc}
0 & 1 \\
-1 & 0
\end{array}\right)
$$

where the indices take values 0 and 1 . With the help of $\epsilon$ one can define the dual space $\mathcal{S}^{*}$ to $\mathcal{S}$ :

$$
\mathcal{S}^{*} \ni z_{A}=z^{B} \epsilon_{B A}, \quad z^{B} \in \mathcal{S} .
$$

There are other complex spaces associated to $\mathcal{S}$ : complex conjugate space $\overline{\mathcal{S}}$ and dual conjugate space $\overline{\mathcal{S}}^{*}$. To distinguish between elements of these spaces we must introduce another type of indices (not only upper and lower), namely primed indices taking value as well 0 or 1 .

$$
z^{A} \in \mathcal{S}, z_{A} \in \mathcal{S}^{*}, \bar{z}^{A^{\prime}} \in \overline{\mathcal{S}}, \bar{z}_{A^{\prime}} \in \overline{\mathcal{S}}^{*}
$$

The symplectic forms in $\mathcal{S}^{*}, \overline{\mathcal{S}}$ and $\overline{\mathcal{S}}^{*}$ are $\epsilon^{A B}, \epsilon_{A^{\prime} B^{\prime}}$ and $\epsilon^{A^{\prime} B^{\prime}}$, which are equal to

$$
\epsilon_{A^{\prime} B^{\prime}}=\epsilon^{A B}=\epsilon^{A^{\prime} B^{\prime}}=\left(\begin{array}{cc}
0 & 1 \\
-1 & 0
\end{array}\right)
$$

We can lower and lift indices with help of $\epsilon$ as follows (since $\varepsilon^{T} \varepsilon=i d$ ):

$$
z_{B}=z^{A} \epsilon_{A B}, w^{A}=\epsilon^{A B} w_{B}, u_{B^{\prime}}=u^{A^{\prime}} \epsilon_{A^{\prime} B^{\prime}}, v^{A^{\prime}}=\epsilon^{A^{\prime} B^{\prime}} v_{B^{\prime}}
$$

One can define isomorphism of the Minkowski space $\mathbb{R}^{1,3}$ and the subspace of $\mathcal{S} \otimes \overline{\mathcal{S}}$ represented by Hermitian matrices. This can be done by taking a distinguished basis of the Hermitian matrices to be the $\sigma_{\alpha}^{A A^{\prime}}$ Pauli matrices:

$$
\begin{aligned}
\sigma_{0}{ }^{A A^{\prime}} & =\frac{1}{\sqrt{2}}\left(\begin{array}{ll}
1 & 0 \\
0 & 1
\end{array}\right), \sigma_{1}{ }^{A A^{\prime}}=\frac{1}{\sqrt{2}}\left(\begin{array}{ll}
0 & 1 \\
1 & 0
\end{array}\right) \\
\sigma_{2}{ }^{A A^{\prime}} & =\frac{1}{\sqrt{2}}\left(\begin{array}{cc}
0 & i \\
-i & 0
\end{array}\right), \sigma_{3}{ }^{A A^{\prime}}=\frac{1}{\sqrt{2}}\left(\begin{array}{cc}
1 & 0 \\
0 & -1
\end{array}\right) .
\end{aligned}
$$

and identify it with the standard basis of the Minkowski space $\mathbb{R}^{1,3}$. This way the element $x=\left(x^{0}, x^{1}, x^{2}, x^{3}\right) \in \mathbb{R}^{1,3}$ is identified with the element

$$
x^{A A^{\prime}}=x^{\alpha} \sigma_{\alpha}^{A A^{\prime}} .
$$

One can read more on spinors in [14] or [9]. 
Since $p \wedge u$ is antisymmetric, one can write it with help of spinors (see [2], [14]) as:

$$
\begin{aligned}
(p \wedge u)^{\alpha \beta} \sigma_{\alpha}{ }^{A A^{\prime}} \sigma_{\beta}{ }^{B B^{\prime}} & =p^{A A^{\prime}} u^{B B^{\prime}}-u^{A A^{\prime}} p^{B B^{\prime}} \\
& =z^{(A} w^{B)} \epsilon^{A^{\prime} B^{\prime}}+\bar{z}^{\left(A^{\prime}\right.} \bar{w}^{\left.B^{\prime}\right)} \epsilon^{A B}
\end{aligned}
$$

for some spinors $z, w \in \mathbb{C}^{2}$, satisfying

$$
z^{(A} w^{B)}=\frac{1}{2}(p \wedge u)_{C^{\prime}}^{A B C^{\prime}} .
$$

It is important to note, that the spinors $z, w$ are given by $p \wedge u$ up to the equivalence class $(z, w) \sim\left(\lambda z, \lambda^{-1} w\right)$, for any nonzero $\lambda \in \mathbb{C}$. One can check, using simple algebra, that

$$
\begin{aligned}
\langle p \wedge u, p \wedge u\rangle & =-\frac{1}{2}\left(z^{(A} w^{B)} \epsilon^{A^{\prime} B^{\prime}}+\bar{z}^{\left(A^{\prime}\right.} \bar{w}^{\left.B^{\prime}\right)} \epsilon^{A B}\right)\left(z_{(A} w_{B)} \epsilon_{A^{\prime} B^{\prime}}+\bar{z}_{\left(A^{\prime}\right.} \bar{w}_{\left.B^{\prime}\right)} \epsilon_{A B}\right) \\
& =4\left(z_{A} w^{A}\right)^{2}=\left(\frac{s}{\pi}\right)^{2}
\end{aligned}
$$

This way, one can set $z_{A} w^{A}= \pm s / 2 \pi$ using the orbit invariant $s$. The meaning of the plus-minus sign will be discussed shortly. It is also possible to show that one can express $w^{A}$ as

$$
w^{A}=p^{A A^{\prime}} \bar{z}_{A^{\prime}}
$$

This, however, is not preserved within an equivalence class $(z, w) \sim\left(\lambda z, \lambda^{-1} w\right)$. This relation restricts the equivalence $(z, w) \sim\left(\lambda z, \lambda^{-1} w\right)$ to $(z, w) \sim\left(e^{i \phi} z, e^{-i \phi} w\right)$ for some real $\phi$. Using the expression $w^{A}=p^{A A^{\prime}} \bar{z}_{A^{\prime}}$, one can see that for $p=$ $( \pm m, 0,0,0)$ one has to choose the sing in $z_{A} w^{A}= \pm s / 2 \pi$ to be the same as the sign of $p^{0}$. To conclude, one can write the orbit given by $p^{2}=m^{2}$ and $\langle p \wedge u, p \wedge u\rangle=(s / \pi)^{2}$ as

$$
\mathcal{O}=\left\{\left(x^{\alpha}, p^{\beta}, z^{A}\right) \mid p^{2}=m^{2}, z_{A} p^{A A^{\prime}} \bar{z}_{A^{\prime}}= \pm \frac{s}{2 \pi}\right\} / \sim
$$

where the equivalence relation $\sim$ is given as

$$
\left(x^{\alpha}, p^{\beta}, z^{A}\right) \sim\left(x^{\alpha}+t p^{\alpha}, p^{\beta}, e^{i \phi} z^{A}\right)
$$

and the plus-minus sign corresponds to different connected components of the orbit. One has to still determine the coadjoint action of the Poincaré group in these new coordinates.

3.1. Action of the Lorentz group on spinors. To determine the action of the Lorentz group on spinors, one has to investigate how the action changes the spinorial version $p^{A A^{\prime}}=p^{\alpha} \sigma_{\alpha}^{A A^{\prime}}$ of a vector $p^{\alpha}$ from the Minkowski space. The determinant of $p^{A A^{\prime}}$ is unchanged under the action of the Lorentz group on $p^{\alpha}$, since $\operatorname{det} p^{A A^{\prime}}=p^{2}$. The multiplication $L_{B}^{A} \bar{L}_{B^{\prime}}^{A^{\prime}} p^{B B^{\prime}}$ of $p^{B B^{\prime}}$ by matrices $L_{B}^{A} \in S l(2, \mathbb{C})$ and $\bar{L}_{B^{\prime}}^{A^{\prime}}=\overline{\left(L_{B}^{A}\right)}$ clearly preserves the determinant of $p^{A A^{\prime}}$ and $L_{B}^{A} \bar{L}_{B^{\prime}}^{A^{\prime}} p^{B B^{\prime}}$ is Hermitian. This way $\pm L$ correspond to some Lorentz transformation and one gets two to one correspondence between $S l(2, \mathbb{C})$ and the Lorentz group. 
Since the Lorentz group acts on the spinor version of $p$ as $\tilde{p}^{A A^{\prime}}=L_{B}^{A} \bar{L}_{B^{\prime}}^{A^{\prime}} p^{B B^{\prime}}$ and it can be shown that $p^{A A^{\prime}}= \pm 2 \pi / s\left(w^{A} \bar{w}^{A^{\prime}}+\left(p^{2} / 2\right) z^{A} \bar{z}^{A^{\prime}}\right)$, one infers that the action of the Lorentz group on spinors is

$$
\begin{gathered}
z^{A} \longrightarrow L_{B}^{A} z^{B} \\
\bar{z}^{A^{\prime}} \longrightarrow \bar{L}_{B^{\prime}}^{A^{\prime} \bar{z}^{B^{\prime}}}
\end{gathered}
$$

The uncertainty which element of $S L(2, \mathbb{C})$ to choose is absorbed in the equivalence class $z \sim e^{i t} z$. From these relations one can get the transformation rules for the dual spinors:

$$
\begin{aligned}
& \tilde{z}^{A}=L_{B}^{A} z^{B} \\
& \tilde{z}_{A}=\tilde{z}^{B} \epsilon_{B A}=L_{C}^{B} z^{C} \epsilon_{B A}=-\epsilon_{A B} L_{C}^{B} \epsilon^{C D} z_{D}
\end{aligned}
$$

Here $\epsilon$ is the symplectic form on the space of two spinors $\mathcal{S}$ and is used to lift the indices. Thus a spinor $z_{A}$ transforms as:

$$
z_{A} \longrightarrow-\epsilon_{A D} L_{C}^{D} \epsilon^{C B} z_{B}=z_{B}\left(L^{-1}\right)^{B}
$$

3.2. Tangent vectors. The tangent vectors generated by the infinitesimal action of $\xi=(A, a) \in \mathfrak{g}$ on the space $\mathbb{R}^{1,3} \times \mathbb{R}^{1,3} \times \mathcal{S}$ are

$$
X_{\xi}(\alpha)=\left(A_{\beta}^{\alpha} x^{\beta}+a^{\alpha}\right) \partial_{x^{\alpha}}+A_{\delta}^{\gamma} p^{\delta} \partial_{p^{\gamma}}+A_{B}^{A} z^{B} \partial_{z^{A}}+\bar{A}_{B^{\prime}}^{A^{\prime}} \bar{z}^{B^{\prime}} \partial_{\bar{z}^{A^{\prime}}}
$$

where $\alpha=(x, p, z), A_{B}^{A}=A_{\beta}^{\alpha} \sigma_{\alpha}^{A A^{\prime}} \sigma_{B A^{\prime}}^{\beta}$ and the action of the restricted Poincaré group is given by

$$
\rho(\Lambda, l) \alpha=(\Lambda x+l, \Lambda p, \pm L z)
$$

with $\pm L$ being elements of $S l(2, \mathbb{C})$ corresponding to $\Lambda$.

Similarly, for the space $\mathbb{R}^{1,3} \times \mathbb{R}^{1,3} \times \overline{\mathcal{S}}^{*}$ the tangent vectors corresponding to the action

are given by

$$
\rho(\Lambda, l) \alpha=\left(\Lambda x+l, \Lambda p, \pm\left(\bar{L}^{T}\right)^{-1} \bar{z}\right)
$$

$$
X_{\xi}(\alpha)=\left(A_{\beta}^{\alpha} x^{\beta}+a^{\alpha}\right) \partial_{x^{\alpha}}+A_{\delta}^{\gamma} p^{\delta} \partial_{p^{\gamma}}+\bar{A}_{A^{\prime}}^{B^{\prime}} \bar{z}_{B^{\prime}} \partial_{\bar{z}_{A^{\prime}}}+A_{A}^{B} z_{B} \partial_{z_{A}}
$$

3.3. Discrete symmetries. To determine the action of the full Poincaré group on spinors, one must find the parity $P$ and time reversal $T$ transformations. From the action of the parity transformation on the Minkowski space

$$
P:\left(u_{0}, u_{1}, u_{2}, u_{3}\right) \rightarrow\left(u_{0},-u_{1},-u_{2},-u_{3}\right)
$$

one can find the following coordinate expression:

$$
(P(u))^{A A^{\prime}}=u_{A^{\prime} A}
$$

Similarly for the time reversal

$$
T:\left(u_{0}, u_{1}, u_{2}, u_{3}\right) \rightarrow-\left(u_{0},-u_{1},-u_{2},-u_{3}\right)
$$

the following expression holds: 


$$
(T(u))^{A A^{\prime}}=-u_{A^{\prime} A}
$$

This suggests

$$
\begin{aligned}
& P:\left(x, p, z^{A}\right) \rightarrow\left(P x, P p, \eta_{P} \bar{z}_{A}\right) \\
& T:\left(x, p, z^{A}\right) \rightarrow\left(T x, T p, \eta_{T} \bar{z}_{A}\right)
\end{aligned}
$$

here $\eta \mathrm{s}$ are some phase factors.

To see that $P$ and $T$ really correspond to antilinear transformations on spinors, one can write the elements of the Lie algebra in a form

$$
A_{\beta}^{\alpha}=\left(\begin{array}{cccc}
0 & x_{1} & x_{2} & x_{3} \\
x_{1} & 0 & y_{3} & y_{2} \\
x_{2} & -y_{3} & 0 & y_{1} \\
x_{3} & -y_{2} & -y_{1} & 0
\end{array}\right)
$$

where $(\vec{x})$ and $(\vec{y})$ are some real vectors. From the relation

$$
A_{B}^{A}=A_{\beta}^{\alpha} \sigma_{\alpha}^{A A^{\prime}} \sigma_{B A^{\prime}}^{\beta}
$$

one obtains

$$
A_{B}^{A}=\left(\begin{array}{cc}
\frac{1}{2} x_{3}-i y_{3} & \frac{1}{2} x_{1}-y_{2}-i\left(\frac{1}{2} x_{2}+y_{1}\right) \\
\frac{1}{2} x_{1}+y_{2}+i\left(\frac{1}{2} x_{2}-y_{1}\right) & -\frac{1}{2} x_{3}+i y_{3}
\end{array}\right) .
$$

Moreover, the adjoint action $G A G^{-1}$ of $g$ on $A \in \mathfrak{g}$ is given in the matrix form for the time reversal $T=\left(\begin{array}{cccc}-1 & 0 & 0 & 0 \\ 0 & 1 & 0 & 0 \\ 0 & 0 & 1 & 0 \\ 0 & 0 & 0 & 1\end{array}\right)$ as

$$
\left(T A T^{-1}\right)^{\alpha}{ }_{\beta}=\left(\begin{array}{cccc}
0 & -x_{1} & -x_{2} & -x_{3} \\
-x_{1} & 0 & y_{3} & y_{2} \\
-x_{2} & -y_{3} & 0 & y_{1} \\
-x_{3} & -y_{2} & -y_{1} & 0
\end{array}\right)_{\beta}^{\alpha}
$$

or

$$
\begin{aligned}
T X_{a} T^{-1} & =-X_{a} \\
T Y_{a} T^{-1} & =Y_{a}
\end{aligned}
$$

where $X_{a}$ is the matrix corresponding to $x_{a}=1$ and all other elements zero. $Y_{a}$ denotes similar matrix with $y_{a}=1$ and all other elements zero. Since for the spinor equivalents $\mathbf{X}_{a}, \mathbf{Y}_{a}$ of $X_{a}, Y_{a}$ one gets the same relations

$$
\begin{aligned}
& T \mathbf{X}_{a} T^{-1}=-\mathbf{X}_{a} \\
& T \mathbf{Y}_{a} T^{-1}=\mathbf{Y}_{a},
\end{aligned}
$$

and moreover $\mathbf{X}_{a} \stackrel{\mathbb{R}}{\sim} i \mathbf{Y}_{a}$, the time reversal acts on spinors as an antilinear operator. Since $P T=-i d$, the parity is antilinear as well. 
3.4. The Orbit. To conclude the above discussion, the orbit corresponding to $m$ and $s$ can be represented as

$$
\mathcal{O}=\left\{\left(x^{\alpha}, p^{\beta}, z^{A}\right) \mid p^{2}=m^{2}, z_{A} p^{A A^{\prime}} \bar{z}_{A^{\prime}}=\frac{ \pm m s}{\sqrt{2}}\right\} / \sim
$$

where the equivalence relation $\sim$ is

$$
z^{A} \sim e^{i t} z^{A}, x \sim x+t^{\prime} p
$$

for some real $t$ and $t^{\prime}$. The coadjoint action of the Poincaré group on the orbit is given by the formulas:

$$
\begin{aligned}
& \rho(\Lambda, l)(x, p, z)=(\Lambda x+l, \Lambda p, \pm L z) \\
& \rho(P)\left(x, p, z^{A}\right)=\left(P x, P p, \eta_{P} \bar{z}_{A}\right) \\
& \rho(T)\left(x, p, z^{A}\right)=\left(T x, T p, \eta_{T} \bar{z}_{A}\right) .
\end{aligned}
$$

We see that the factorization by the equivalence classes reduces the sign ambiguity in $\pm L$ corresponding to $\Lambda$ as well as the phase factors $\eta_{P}$ and $\eta_{T}$.

3.5. Coordinates. One can choose local coordinate systems on the orbit $\mathcal{O}$ to be

$$
\phi_{1}(x, p, z)=\left(x_{1}, x_{2}, x_{3}, p_{1}, p_{2}, p_{3}, \frac{z_{0}}{z_{1}}\right)
$$

on the open set $U_{1}=\left\{z_{1} \neq 0\right\}$, and

$$
\phi_{0}(x, p, z)=\left(x_{1}, x_{2}, x_{3}, p_{1}, p_{2}, p_{3}, \frac{z_{1}}{z_{0}}\right)
$$

on the open set $U_{0}=\left\{z_{0} \neq 0\right\}$. It is convenient to introduce new coordinates on each of these coverings, namely $c_{0}=\frac{z_{1}}{z_{0}}$ on $U_{0}$ and $c_{1}=\frac{z_{0}}{z_{1}}$ on $U_{1}$.

On the overlap of these two sets the transition function $\phi_{0} \phi_{1}^{-1}$ is

$$
\phi_{0} \phi_{1}^{-1}\left(x_{1}, x_{2}, x_{3}, p_{1}, p_{2}, p_{3}, c_{1}\right)=\left(x_{1}, x_{2}, x_{3}, p_{1}, p_{2}, p_{3}, \frac{1}{c_{1}}\right),
$$

Similarly

$$
\phi_{1} \phi_{0}^{-1}\left(x_{1}, x_{2}, x_{3}, p_{1}, p_{2}, p_{3}, c_{0}\right)=\left(x_{1}, x_{2}, x_{3}, p_{1}, p_{2}, p_{3}, \frac{1}{c_{0}}\right)
$$

The last component transforms according to the transition function for a sphere in stereographic coordinates. Thus one can conclude that

$$
\mathcal{O}=T P \times S^{2},
$$

where $T P$ is the tangent bundle of two-sheeted hyperboloid given by $p^{2}=m^{2}$, since one can choose a representant of the equivalence class $x \sim x+t p$ to be such $x$ that $p x=0$. 
3.6. Symplectic structure. One wants to find the symplectic structure on the orbit $\mathcal{O}$. To do so, the following theorem proved in [14] can be applied.

Theorem 2. Let $(\mathcal{O}, \omega)$ be a coadjoint orbit of $G$ and $W$ a manifold where $G$ acts as $\mathfrak{g} \ni \xi \rightarrow X_{\xi}^{\prime}$ with a surjection $\pi: W \rightarrow \mathcal{O}$ and $\theta$ is a one form on $W$ such

- $\pi^{-1}(\alpha)$ is connected for all $\alpha \in \mathcal{O}$

- $\pi_{*} X_{\xi}^{\prime}=X_{\xi}$

- for all $x \in W$ the following holds $\left.X_{\xi}^{\prime}\right\lrcorner \theta(x)=\alpha(\xi)$ where $\alpha=\pi(x) \in \mathcal{O} \subset$ $\mathfrak{g}^{*}$

then $\theta$ is invariant under action of $G$ and $d \theta=\pi^{*} \omega$.

Since one can consider the surjection $\pi: W \rightarrow \mathcal{O}$ to be the equivalence class 8 identification in $W=\left\{(x, p, z) \in \mathbb{R}^{1,3} \times \mathbb{R}^{1,3} \times \mathbb{C}^{2}\right\}$, one can use the above theorem provided that there is a 1 -form $\theta$ on $W$ such that

$$
\theta_{\alpha}\left(X_{\xi}\right)=\alpha(\xi) \text {. }
$$

Since

$$
\begin{aligned}
\alpha(\xi) & =\left\langle p \wedge x+^{*}(p \wedge u), A\right\rangle-\langle p, a\rangle \\
& =-\langle p, A x+a\rangle-\frac{1}{2} i\left(z^{(A} w^{B)} \epsilon^{A^{\prime} B^{\prime}}-\bar{z}^{\left(A^{\prime}\right.} \bar{w}^{\left.B^{\prime}\right)} \epsilon^{A B}\right) A_{A B A^{\prime} B^{\prime}} \\
& =-p_{\alpha} d x^{\alpha}\left(X_{\xi}\right)-\frac{i}{2}\left(z^{(A} w^{B)} A_{A B A^{\prime}}{ }^{\prime}-\bar{z}^{\left(A^{\prime}\right.} \bar{w}^{\left.B^{\prime}\right)} A_{A}^{A} A^{\prime} B^{\prime}\right) \\
& =-p_{\alpha} d x^{\alpha}\left(X_{\xi}\right)+i\left(z^{A} A_{A B} w^{B}-\bar{z}^{A^{\prime}} \bar{A}_{A^{\prime} B^{\prime}} \bar{w}^{B^{\prime}}\right) \\
& =-p_{\alpha} d x^{\alpha}\left(X_{\xi}\right)+i\left(d z_{B}\left(X_{\xi}\right) w^{B}-d \bar{z}_{B^{\prime}}\left(X_{\xi}\right) \bar{w}^{B^{\prime}}\right),
\end{aligned}
$$

using the expression (7) for $X_{\xi}$ and the formula (see [14])

$$
*(p \wedge u)=i\left(z^{(A} w^{B)} \epsilon^{A^{\prime} B^{\prime}}-\bar{z}^{\left(A^{\prime}\right.} \bar{w}^{\left.B^{\prime}\right)} \epsilon^{A B}\right) .
$$

Then

$$
\theta=-i p_{A A^{\prime}}\left(z^{A} d \bar{z}^{A^{\prime}}-\bar{z}^{A^{\prime}} d z^{A}\right)-p_{\alpha} d x^{\alpha}
$$

defines a symplectic potential on $\mathcal{O}$. The symplectic form on the orbit is $\omega=\mathrm{d} \theta$. Note, that by this definition $\theta$ is well defined as an element of $T^{*} \mathcal{O}$, since there is a difference of the complex conjugate elements multiplied by $i$, or as an element of the complexified space $T_{\mathbb{C}}^{*} \mathcal{O}$.

3.7. Hamiltonian vector fields. The Hamiltonian vector field is defined as

$$
\left.X_{f}\right\lrcorner \omega=-d f
$$

One can easily verify that the Hamiltonian field for the function $p^{2}$ is

$$
X_{p^{2}}=-2 p \partial_{x}
$$


Similarly for the function $z^{A} p_{A A^{\prime}} \bar{z}^{A^{\prime}}$ (denoted $z p \bar{z}$ ) one gets the Hamiltonian vector field

$$
X_{z p \bar{z}}=-\frac{i}{2}\left(z^{A} \partial_{z^{A}}-\bar{z}^{A^{\prime}} \partial_{\bar{z}^{A^{\prime}}}\right)
$$

because the flow of this field is $\left(e^{-i t / 2} z^{A}, e^{i t / 2} \bar{z}^{A^{\prime}}\right)$ and it preserves $\theta$ (i.e. $£_{X_{z p \bar{z}}} \theta=$ $0)$. Thus $\left.\left.\left.X_{z p \bar{z}}\right\lrcorner \omega=£_{X_{z p \bar{z}}} \theta-d\left(X_{z p \bar{z}}\right\lrcorner \theta\right)=-d\left(X_{z p \bar{z}}\right\lrcorner \theta\right)=-d\left(z^{A} p_{A A^{\prime}} \bar{z}^{A^{\prime}}\right)$. Again, even though the vector $X_{z p \bar{z}}$ formally belongs to the complexified tangent space, it is an element of the real tangent space, since it is a difference of the complex conjugate vectors multiplied by $i$. Note also, that these vector fields are well defined as both elements of $T \mathcal{O}$ and $T W$.

\section{Quantization}

4.1. Prequantization. The purpose of prequantization is to construct a faithful representation of the algebra of the smooth functions on a symplectic manifold $(M, \omega)$ with the bracket operation being the Poisson bracket given by the symplectic structure on $M$. To do this, one can consider a line bundle over $M$. To be able to differentiate the sections of this bundle, one must introduce a connection. If the curvature of the connection is equal to the symplectic form, the line bundle together with the connection is called the prequantum bundle. There is a necessary condition on the curvature to construct a line bundle with this connection. This condition is generally referred to as the integrality condition (see e.g. [7]):

Condition 1. The integral over any closed oriented 2-surface in $M$ of the curvature form is a natural number.

In the case of the symplectic form given by the potential (9), the integrality condition implies that $2 s \in \mathbb{N}$.

From now on, only the trivial line bundle is considered. To define a scalar product on the space of the sections $\lambda$, one can set

$$
\left(\lambda_{1}, \lambda_{2}\right)=\int_{\mathcal{O}}\left(\lambda_{1}, \lambda_{2}\right)_{m} \omega^{n}
$$

Here $\left(\lambda_{1}, \lambda_{2}\right)_{m}$ denotes the scalar product of $\lambda_{1}(m)$ and $\lambda_{2}(m)$ in the fibre $\pi^{-1}(m)$, where $m \in \mathcal{O}$. This integral thus defines a scalar product and the space $\mathcal{H}$ of the square integrable sections.

The trivializing section $\lambda_{0}$ is defined so that

$$
\nabla \lambda_{0}=2 \pi i \theta \otimes \lambda_{0} .
$$

Note that here $\lambda_{0}$ is defined up to a constant multiplier. One can compute Hermitian structure (.,.) as

$$
d\left(\lambda_{0}, \lambda_{0}\right)=\left(\nabla \lambda_{0}, \lambda_{0}\right)+\left(\lambda_{0}, \nabla \lambda_{0}\right)=2 \pi i(\theta-\bar{\theta})\left(\lambda_{0}, \lambda_{0}\right)=0
$$

Thus one can set $\left(\lambda_{0}, \lambda_{0}\right)=1$. 
The prequantized operator $P_{\xi}$ corresponding to the generator $\xi \in \mathfrak{g}$ acts on a section $\lambda=\psi \lambda_{0}$ as

$$
\begin{aligned}
P_{\xi}\left(\psi \lambda_{0}\right) & =\left(\nabla_{X_{\xi}}-2 \pi i J_{\xi}\right)\left(\psi \lambda_{0}\right) \\
& =\left(\nabla_{X_{\xi}}-2 \pi i \theta\left(X_{\xi}\right)\right)\left(\psi \lambda_{0}\right) \\
& =\left(X_{\xi} \psi+2 \pi i \theta\left(X_{\xi}\right) \psi-2 \pi i \theta\left(X_{\xi}\right) \psi\right) \lambda_{0} \\
& =\left(X_{\xi} \psi\right) \lambda_{0},
\end{aligned}
$$

where $J_{\xi}$ is the momentum map ${ }^{2}$ evaluated at $\xi$ and satisfies $J_{\xi}=\theta\left(X_{\xi}\right)$.

The prequantized operator corresponding to the time reversal $T$ (or any other discrete symmetry) can be computed as follows. An action of $T$ on the trivializing section $\lambda_{0}$ can be expressed as

$$
T \lambda_{0}=f \lambda_{0} \circ T
$$

where $f$ is to be determined. This way one can write

$$
T^{*} \nabla \lambda_{0}=\nabla T \lambda_{0}=(d f+2 \pi i f \theta) \otimes \lambda_{0} \circ T
$$

or equivalently

$$
T^{*} \nabla \lambda_{0}=T^{*}\left(2 \pi i \theta \otimes \lambda_{0}\right)=2 \pi i f T^{*} \theta \otimes \lambda_{0} \circ T .
$$

Comparing the right sides of the last expressions and since $T^{*} \theta=\theta$, one infers that $d f=0$, or $f=$ const.. The action of $T$ on an arbitrary section is

$$
T\left(\psi \lambda_{0}\right)=\tilde{\psi} f \lambda_{0} \circ T
$$

where $\tilde{\psi}$ corresponds to the transformed $\psi$. Using the fact that $T$ commutes with the connection, one can that

$$
T\left(\nabla \psi \lambda_{0}\right)=\nabla\left(\tilde{\psi} f \lambda_{0} \circ T\right)=f d \tilde{\psi} \otimes \lambda_{0} \circ T+2 \pi i f \tilde{\psi} \theta \otimes \lambda_{0} \circ T,
$$

The above can be expressed also as

$T\left(\nabla \psi \lambda_{0}\right)=T\left(d \psi \otimes \lambda_{0}+2 \pi i \psi \theta \otimes \lambda_{0}\right)=\left(T^{*} d \psi\right) \otimes f \lambda_{0} \circ T+2 \pi i \tilde{\psi} T^{*} \theta \otimes f \lambda_{0} \circ T$.

From this follows that $T^{*} d \psi \equiv d T^{*} \psi=d \tilde{\psi}$, or

$$
T \psi=\tilde{\psi}=T^{*} \psi+\text { const. }=\psi \circ T+\text { const. }
$$

If one requires that $T$ preserve the norm, one gets

$$
T \psi=\psi \circ T
$$

and $f=1$. By similar reasoning, one can write that

$$
P \psi=\psi \circ P .
$$

\footnotetext{
${ }^{2}$ More on momentum maps can be found in [1]
} 
4.2. Polarization. To quantize the prequantum line bundle, one has to introduce a polarization and then reduce the prequantum bundle to the sections that are constant along the introduced polarization. The spinorial part of the orbit is diffeomorphic to a sphere. On a sphere, however, all the polarizations have nonzero real and imaginary components. The parity $P$ is an antilinear map, and thus does not preserve these polarizations. There is no invariant polarization under the full Poincaré group on the orbit $\mathcal{O}$. There is no problem in studying only the irreducible representations of the restricted Poincaré group, i.e. the connected component of the Poincaré group, since the polarization is invariant on the connected component $\mathcal{O}_{0}$ of the orbit $\mathcal{O}$, since $\mathcal{O}_{0}$ is the orbit of the restricted Poincaré group. One can choose to work with the component corresponding to $p_{0}>0$. To recall, this orbit is given by

$$
\mathcal{O}_{0}=\left\{(x, p, z) \mid p^{2}=m^{2}, z p \bar{z}=\frac{s}{2 \pi}\right\} /\left(x \sim x+t p, z \sim e^{i t^{\prime}} z\right) .
$$

The condition $p_{0}>0$ is implicitly included in the constraint $z p \bar{z}=s / 2 \pi$. The line bundle over this orbit can be constructed as a line bundle over one connected component of the constraint manifold

$$
C=\left\{\left(x, p, z^{A}\right) \mid p^{2}=m^{2}, z p \bar{z}=\frac{s}{2 \pi}\right\}
$$

with the following restriction on the sections

$$
\begin{aligned}
\nabla_{X_{p^{2}}} \lambda & =0 \\
\nabla_{X_{z p p z}} & =0
\end{aligned}
$$

where $\lambda$ is a section over $C$ and $X_{f}$ is a restriction of the Hamiltonian vector field corresponding to the function $f$ in $M$ to $C$.

The flow of $X_{p^{2}}$ is $(x, p, z) \mapsto(x-2 t p, p, z)$ and the flow of $X_{z p \bar{z}}$ is $(x, p, z) \mapsto$ $\left(x, p, \exp \left(-\frac{i}{2} t\right) z\right)$. These flows are considered as flows of restrictions of the Hamiltonian vector fields on $W$ to $C$. Here one replaces the factorization of the constraint space $C$ by the equivalence relations with the requirement of the sections to be constant along the flows given by the factorizing equivalence relations. One can make this construction, since the flow of $X_{p^{2}}$ is not closed and the parallel transport along the field $X_{z p \bar{z}}$ changes the phase by factor $\exp \left(2 \pi \int \theta\right)$, what, by the quantization condition, is equal to $\exp (4 \pi s)=1$. The equivalence (10) can be written as

$$
\begin{aligned}
p \partial_{x} \psi & =2 \pi i p^{2} \psi=2 \pi i m^{2} \psi \\
\left(z \partial_{z}-\bar{z} \partial_{\bar{z}}\right) \psi & =4 \pi z p \bar{z} \psi= \pm 2 s \psi
\end{aligned}
$$

where $\lambda=\psi \lambda_{0}$.

The problem with this construction is that $C$ together with $\omega$ extended to it is a presymplectic manifold and thus one can not use the quantization theory on this space. There is, however, a way around this problem by considering commutation of reduction and quantization (see [6] and the references therein) and taking the 
space $W$. One can construct a line bundle over this space and restrict the sections of this line bundle to sections satisfying

$$
\begin{aligned}
\nabla_{X_{p^{2}}} \lambda & =0 \\
\nabla_{X_{z p \bar{z}}} & =0
\end{aligned}
$$

as before, plus condition that restricts the support of the sections to the constraint manifold $C$

$$
\begin{aligned}
P_{p^{2}-m^{2}} \lambda & =0 \\
P_{z p \bar{z}-s / 2 \pi} \lambda & =0 .
\end{aligned}
$$

Here $P$ s are the prequantized operators corresponding to the indicated functions. To see how these operators restrict the support of the sections, one expresses the formulas explicitly as

$$
\begin{aligned}
P_{p^{2}-m^{2}} \lambda & =\nabla_{X_{p^{2}}} \lambda-2 \pi i\left(p^{2}-m^{2}\right) \lambda=0 \\
P_{z p \bar{z}-s / 2 \pi} \lambda & =\nabla_{X_{z p \bar{z}}} \lambda-2 \pi i\left(z p \bar{z}-\frac{s}{2 \pi}\right) \lambda=0
\end{aligned}
$$

which with (11) yields that the support of a section is given by equations $p^{2}=m^{2}$ and $z p \bar{z}=s / 2 \pi$.

The distribution given by $\left\{\partial_{\bar{c}}\right\}_{\mathbb{C}}=\left\{\bar{w} \partial_{\bar{z}}\right\}_{\mathbb{C}}$ is well defined $\left(\partial_{\bar{c}}\right.$ is a well defined complex polarization on a sphere) and is invariant under restricted Poincaré group since it is a restriction of $\left\{\partial_{\bar{z}}\right\}_{\mathbb{C}}$ to $C,\left[\partial_{\bar{z}}, X_{\xi}\right] \in \partial_{\bar{z}}$ and $C$ is an invariant manifold. This way one can define a polarization on the orbit of the restricted Poincaré group corresponding to $m$ and $s$ and denoted by $\mathcal{O}_{0}$ as

$$
F_{\mathcal{O}_{0}}=\left\{u \partial_{x}, \bar{w} \partial_{\bar{z}}\right\}_{\mathbb{C}}
$$

where $u$ is such, that $u \partial_{x} \in T \mathcal{O}_{0}$. It is important that $F_{\mathcal{O}_{0}}$ is restriction of

$$
F=\left\{\partial_{x}, \partial_{\bar{z}}\right\}_{\mathbb{C}} \subset T \mathbb{R}^{1,3} \times \mathcal{S}=W
$$

to the orbit $\mathcal{O}_{0}$.

This way, one can represent the line bundle over the orbit $\mathcal{O}_{0}$ as the line bundle over $T \mathbb{R}^{1,3} \times \mathcal{S}$ such that

$$
\left\{\lambda \mid \nabla_{X_{p^{2}}} \lambda=0, \nabla_{X_{z p \bar{z}}} \lambda=0, P_{p^{2}-m^{2}} \lambda=0, P_{z p \bar{z}-s / 2 \pi} \lambda=0\right\}
$$

and define the polarization to be

$$
F=\left\{\partial_{x}, \partial_{\bar{z}}\right\}_{\mathbb{C}} .
$$

4.2.1. Quantization. One takes the prequantum space

$$
H=\left\{\lambda \mid \nabla_{X_{p^{2}}} \lambda=0, \nabla_{X_{z p \bar{z}}} \lambda=0, P_{p^{2}-m^{2}} \lambda=0, P_{z p \bar{z}-s / 2 \pi} \lambda=0\right\}
$$

and restricts it to the sections that are covariantly constant along the polarization. The restriction of $H$ to the sections covariantly constant along the polarization 
$F=\left\{\partial_{x}, \partial_{\bar{z}}\right\}_{\mathbb{C}}$ will be denoted $H_{p}$. Since the polarization $F$ contains vector $X_{p^{2}}$, one can write the quantized space as

$$
H_{p}=\left\{\lambda \mid \nabla_{\partial_{x}} \lambda=0, \nabla_{\partial_{\bar{z}}} \lambda=0, \nabla_{X_{z p \bar{z}}} \lambda=0, P_{p^{2}-m^{2}} \lambda=0, P_{z p \bar{z}-s / 2 \pi} \lambda=0\right\} .
$$

For the polarization $F=\left\{\partial_{x}, \partial_{\bar{z}}\right\}_{\mathbb{C}}$ one writes the quantization restriction on sections as

$$
0=\nabla_{F} \lambda=(2 \pi i \theta(F)+F) \psi \otimes \lambda_{0}
$$

or

$$
\begin{aligned}
\partial_{\bar{z}} \psi & =0 \\
\partial_{x} \psi & =2 \pi i p \psi
\end{aligned}
$$

After setting $\nabla_{X_{z p \bar{z}}} \lambda=0$, one obtains

$$
\left(z \partial_{z}-\bar{z} \partial_{\bar{z}}\right) \psi=4 \pi z p \bar{z} \psi
$$

This can be written with help of the constraint $(z p \bar{z}-m s / \sqrt{2}) \lambda=0$ on the support of $\lambda$ and the requirement of covariant constancy along the polarization as

$$
z \partial_{z} \psi=2 s \psi
$$

From the integrality condition 1 follows that $2 s \in \mathbb{N}$. Thus, after taking into account that the sections must be constant also along $\partial_{x}$, one can express the elements of $H_{p}$ as

$$
\psi=e^{2 \pi i\langle p, x\rangle} z^{A_{1}} z^{A_{2}} \ldots z^{A_{2 s}} \xi_{A_{1} A_{2} \ldots A_{2 s}}(p) .
$$

One wants to determine the action of the quantized operator $U_{\xi}^{t}$ corresponding to the one parameter subgroups generated by $\xi \in \mathfrak{g} .^{3}$

$$
U_{\xi}^{t} \lambda=\left(\phi_{t}^{\#}\right)^{-1} \circ \lambda \circ \phi_{t}
$$

where $\phi_{t}$ is the flow of $X_{\xi}$ and $\phi_{t}^{\#}$ is the flow of the lifted vector field $Z_{\xi}=$ $\left.\frac{\mathrm{d}}{\mathrm{d} t} e^{2 \pi i J_{m}(\xi)} \lambda(m)\right|_{t=0}$ of $X_{\xi}$ to the line bundle. For a general section $\psi \lambda_{0}$ one gets

$$
\begin{aligned}
U_{\xi}^{t} \lambda & =\left(\phi_{t}^{\#}\right)^{-1} \circ \lambda \circ \phi_{t}=\phi_{-t}^{\#}\left(\left(\psi \circ \phi_{t}\right) \lambda_{0} \circ \phi_{t}\right) \\
& =\left(\psi \circ \phi_{t}\right) \phi_{-t}^{\#} \circ \lambda_{0} \circ \phi_{t}
\end{aligned}
$$

The flow of $Z_{\xi}$ can be split into the horizontal (parallel) and vertical parts. The parallel section $\psi \lambda_{0}$ along a path $\phi_{t}$ is given by ${ }^{4}$

$$
\psi(c(t))=\psi_{0} e^{-2 \pi i \int_{\phi_{t}} \theta} .
$$

\footnotetext{
${ }^{3}$ More details can be found in [12].

${ }^{4} 0=\nabla_{X} \lambda=(X \psi+2 \pi i \theta(X) \psi) \lambda_{0}$

$X \psi=-2 \pi i \theta(X) \psi$

$\psi=e^{-2 \pi i \int_{\phi_{t}} \theta(X) d t} \psi_{0}$
} 
The vertical part of $\phi_{t}^{\#}$ can be computed as

$$
z \mapsto e^{2 \pi i \int_{\phi_{t}} J_{\xi} \mathrm{d} t}
$$

and hence

$$
U_{\xi}^{t} \lambda=\left(\psi \circ \phi_{t}\right) e^{2 \pi i \int_{\phi_{t}}\left(J_{\xi}-\theta\left(X_{\xi}\right)\right) \mathrm{d} t} \lambda_{0}
$$

where one integrates along the flow $\phi_{t}$ of the vector field $X_{\xi}$. This formula can be written also for the operator corresponding to a function $f$ as

$$
U_{f}^{t} \lambda=\left(\psi \circ \phi_{t}\right) e^{2 \pi i \int_{\phi_{t}}\left(f-\theta\left(X_{f}\right)\right) \mathrm{d} t} \lambda_{0}
$$

where $\phi_{t}$ is in this case the flow of the vector field $X_{f}$.

Since, from the construction of $\theta$, one has that $\theta\left(X_{\xi}\right)(\alpha)=\alpha(\xi)$ and $J_{\xi}(\alpha)=$ $\alpha(\xi)$, one obtains

$$
U_{\xi}^{t} \lambda=\left(\psi \circ \phi_{t}\right) \lambda_{0}
$$

Note that this can not be generalized to the case of an arbitrary function $f$, since, in general, $f \neq \theta\left(X_{f}\right)$.

Note also that considering only sections covariantly constant along the polarization $F$, one looses the scalar product. One can fix this problem by using so called half-densities; here they are, however, not treated and the construction of the scalar product is omitted.

4.2.2. Free Dirac Equation. In the following only the case when $s=1 / 2$ is treated. The action of the discrete symmetries does not preserve the polarization (it is impossible to find a polarization that would be preserved by this action since $T$ and $P$ act as antilinear operators on the spinorial part of the orbit and the only polarization on a sphere is given by $\partial_{z}$ or $\partial_{\bar{z}}$ ). To allow the discrete symmetries, one can extend the Hilbert space in such a way, that the discrete symmetries preserve the polarization. This can be done by inducing the representation of the restricted Poincaré group obtained in the previous section to the representation of the full Poincaré group.

There is a well defined action of the discrete symmetries on the sections of the representation space $H_{p}$ which can be considered as a subspace of $H$. Thus, one can construct three other spaces corresponding to all discrete symmetries

$$
\begin{aligned}
H_{p}^{P} & =P\left(H_{p}\right) \\
H_{p}^{T} & =T\left(H_{p}\right) \\
H_{p}^{T P} & =T P\left(H_{p}\right)
\end{aligned}
$$

The explicit realization of the spaces corresponding to the transformed space

$$
H_{p}=\left\{\lambda \mid \nabla_{\partial_{x}} \lambda=0, \nabla_{\partial_{\bar{z}}} \lambda=0, \nabla_{X_{z p \bar{z}}} \lambda=0, P_{p^{2}-m^{2}} \lambda=0, P_{z p \bar{z}-s / 2 \pi} \lambda=0\right\}
$$


by the discrete symmetries is as follows:

$$
\begin{aligned}
H_{p}^{T P} & =\left\{\lambda \mid \nabla_{\partial_{x}} \lambda=0, \nabla_{\partial_{\bar{z}}} \lambda=0, \nabla_{X_{z p \bar{z}}} \lambda=0, P_{p^{2}-m^{2}} \lambda=0, P_{z p \bar{z}+s / 2 \pi} \lambda=0\right\} \\
H_{p}^{T} & =\left\{\lambda \mid \nabla_{\partial_{x}} \lambda=0, \nabla_{\partial_{z}} \lambda=0, \nabla_{X_{z p \bar{z}}} \lambda=0, P_{p^{2}-m^{2}} \lambda=0, P_{z p \bar{z}+s / 2 \pi} \lambda=0\right\} \\
H_{p}^{P} & =\left\{\lambda \mid \nabla_{\partial_{x}} \lambda=0, \nabla_{\partial_{z}} \lambda=0, \nabla_{X_{z p \bar{z}}} \lambda=0, P_{p^{2}-m^{2}} \lambda=0, P_{z p \bar{z}-s / 2 \pi} \lambda=0\right\}
\end{aligned}
$$

To see why this is so, one can recall the action of the discrete symmetries

$$
\begin{aligned}
& P:\left(x, p, z^{A}\right) \rightarrow\left(P x, P p, \eta_{P} \bar{z}_{A}\right) \\
& T:\left(x, p, z^{A}\right) \rightarrow\left(T x, T p, \eta_{T} \bar{z}_{A}\right)
\end{aligned}
$$

and thus the transformations $P$ and $T$ must map the polarization to its conjugate and the transformations $T$ and $T P$ transform the connected component of $p^{2}=m^{2}$ with $p_{0}>0$ to the connected component with $p_{0}<0$. The typical elements of these spaces are

$$
\psi^{T P}=e^{2 \pi i\langle p, x\rangle} z^{A} \xi_{A}(p)
$$

with the support on $p^{2}=m^{2}, p_{0}<0, z p \bar{z}=s / 2 \pi$

$$
\psi^{T}=e^{2 \pi i\langle p, x\rangle} \bar{z}_{A^{\prime}} \eta^{A^{\prime}}(p)
$$

with the support on $p^{2}=m^{2}, p_{0}<0, z p \bar{z}=-s / 2 \pi$ and

$$
\psi^{P}=e^{2 \pi i\langle p, x\rangle} \bar{z}_{A^{\prime}} \eta^{A^{\prime}}(p)
$$

with the support on $p^{2}=m^{2}, p_{0}>0, z p \bar{z}=-s / 2 \pi$. This way, one can represent all the wave functions as one multivalued function form the space $\mathcal{H}_{p}=H_{p} \oplus H_{p}^{T} \oplus$ $H_{p}^{P} \oplus H_{p}^{T P}$ :

$$
\psi=e^{2 \pi i\langle p, x\rangle}\left(\begin{array}{c}
z^{A} \xi_{A}(p) \\
\bar{z}_{A^{\prime}} \eta^{A^{\prime}}(p)
\end{array}\right)
$$

with the support on the whole $p^{2}=m^{2}$ and $z p \bar{z}=s / 2 \pi$ for the first component and $z p \bar{z}=-s / 2 \pi$ for the second component. The action of the operators $U_{(A, a)}^{t}$ generated by the infinitesimal generators $(A, a)$ is given on each component separately. To determine $U$ one follows the same procedure as in the case of the space $H_{P}$ to find the action on the first component as

$$
U_{(A, a)}^{t} \psi^{+}=e^{2 \pi i\left\langle e^{A t} p, e^{A t} x+a t\right\rangle} e^{A t} z \xi\left(e^{A t} p\right)
$$

This operator acts similarly on the other component as well. After recalling the action of the Lorentz group on dual conjugate spinors one can summarize:

$$
U_{(A, a)}^{t}\left[e^{2 \pi i\langle p, x\rangle}\left(\begin{array}{c}
\xi_{A} \\
\eta^{A^{\prime}}
\end{array}\right)(p)\right]=e^{2 \pi i\langle p, a\rangle}\left(\begin{array}{c}
e^{A^{T} t} \xi_{A} \\
e^{-\bar{A} t} \eta^{A^{\prime}}
\end{array}\right)\left(e^{A t} p\right)
$$

This representation is however not irreducible. Since this construction should correspond to a single spinning particle, one would like to obtain an irreducible representation, as discussed in [8]. There is an operator which commutes with all the 
$U_{(A, a)}^{t}$ and all the discrete symmetries. This operator can be written as

$$
D \psi(x, p, z)=e^{2 \pi i\langle p, x\rangle}\left(\begin{array}{c}
z^{A} p_{A A^{\prime}} \eta^{A^{\prime}}(p) \\
\xi_{A}(p) p^{A A^{\prime}} \bar{z}_{A^{\prime}}
\end{array}\right)
$$

or in the matrix form acting only on the spinor part of the function, given by $\operatorname{vector}\left(\begin{array}{c}\xi_{A} \\ \eta^{A^{\prime}}\end{array}\right)$ :

$$
D=\left(\begin{array}{cc}
0 & p_{A A^{\prime}} \\
\left(p^{T}\right)^{A^{\prime} A} & 0
\end{array}\right)
$$

To show that this operator commutes with both $U$ and the discrete symmetries, one has to determine explicitly the action of the discrete symmetries:

$$
T \psi(x, p, z)=e^{2 \pi i\langle p, x\rangle}\left(\begin{array}{c}
\bar{z}_{A^{\prime}} \eta^{A^{\prime}}(T p) \\
z^{A} \xi_{A}(T p)
\end{array}\right)
$$

since $T$ acts as a pull-back and maps $z^{A}$ to $\bar{z}_{A^{\prime}}$. Similarly

$$
P \psi(x, p, z)=e^{2 \pi i\langle p, x\rangle}\left(\begin{array}{c}
\bar{z}_{A^{\prime}} \eta^{A^{\prime}}(P p) \\
z^{A} \xi_{A}(P p)
\end{array}\right)
$$

One can easily show that

$$
\begin{aligned}
D T \psi(x, p, z) & =D\left(e^{2 \pi i\langle p, x\rangle}\left(\begin{array}{c}
\bar{z}_{A^{\prime}} \eta^{A^{\prime}}(T p) \\
z^{A} \xi_{A}(T p)
\end{array}\right)\right) \\
& =e^{2 \pi i\langle p, x\rangle}\left(\begin{array}{c}
\bar{z}_{A^{\prime}}(T p)^{A A^{\prime}} \xi_{A}(T p) \\
z^{A}(T p)_{A A^{\prime}} \eta^{A^{\prime}}(T p)
\end{array}\right) \\
T D \psi(x, p, z) & =T\left(e^{2 \pi i\langle p, x\rangle}\left(\begin{array}{c}
z^{A} p_{A A^{\prime}} \eta^{A^{\prime}}(p) \\
\xi_{A}(p) p^{A A^{\prime}} \bar{z}_{A^{\prime}}
\end{array}\right)\right) \\
& =e^{2 \pi i\langle p, x\rangle}\left(\begin{array}{c}
\bar{z}_{A^{\prime}}(T p)^{A A^{\prime}} \xi_{A}(T p) \\
z^{A}(T p)_{A A^{\prime}} \eta^{A^{\prime}}(T p)
\end{array}\right)
\end{aligned}
$$

Thus $D T=T D$. Similarly $D P=P D$. To show that $D$ commutes with all $U^{\prime}$, it is enough to prove that $D$ preserves the flow of $X_{\xi}$. Since $D$ acts as a pull-back in the spinorial part of the coordinates

$$
\mathcal{S} \ni z^{A} \stackrel{D}{\longleftrightarrow} z^{A} p_{A A^{\prime}} \in \overline{\mathcal{S}}^{*}
$$


one has to check the action of $D$ only on the spinor part of the wave function

$$
\begin{aligned}
D\left(e^{A^{A}{ }_{B} t} z^{B}\right) & =e^{A_{B}^{A} t} z^{B}\left(e^{A t} p\right)_{A A^{\prime}} \\
& =e^{A^{A}{ }_{B} t} z^{B}\left(p_{C C^{\prime}} e^{-A_{A}^{C} t} e^{-\bar{A}_{A^{\prime}}^{C^{\prime}} t}\right) \\
& =z^{C} p_{C C^{\prime}} e^{-\bar{A}_{A^{\prime}}^{C^{\prime}} t} \\
& =(D z)_{C^{\prime}} e^{-\bar{A}_{A^{\prime}}^{C^{\prime}} t} \\
& =\phi_{\xi}^{t}(D z)
\end{aligned}
$$

Similarly, the dual-conjugate part of the flow is preserved under the action of the operator $D$.

To construct an irreducible representation of the Poincaré group, one must restrict the investigation to a eigenspaces of the operator $D$. Only these subspaces have a chance to form an irreducible representation.

The operator $D$ has an eigenvalue $\pm m$, since

$$
\operatorname{det}\left\{\left(\begin{array}{cc}
0 & p_{A A^{\prime}} \\
\left(p^{T}\right)^{A^{\prime} A} & 0
\end{array}\right)-\lambda I\right\}=\lambda^{4}-p^{4}=\lambda^{4}-m^{4} .
$$

The eigenvector equation

$$
D \psi= \pm m \psi
$$

can be rewritten as

$$
\sqrt{2} p_{\alpha}\left(\begin{array}{cc}
0 & \sigma^{\alpha} A_{A^{\prime}} \\
\sigma^{\alpha A A^{\prime}} & 0
\end{array}\right)\left(\begin{array}{c}
\xi_{A} \\
\eta^{A^{\prime}}
\end{array}\right)= \pm m\left(\begin{array}{c}
\xi_{A} \\
\eta^{A^{\prime}}
\end{array}\right)
$$

To write the above in the matrix multiplication notation, one can transpose the appropriate block as

$$
\sqrt{2} p_{\alpha}\left(\begin{array}{cc}
0 & \sigma^{\alpha}{ }_{A A^{\prime}} \\
\bar{\sigma}^{\alpha A^{\prime} A} & 0
\end{array}\right)\left(\begin{array}{l}
\eta^{A^{\prime}} \\
\xi_{A}
\end{array}\right)= \pm m\left(\begin{array}{l}
\eta^{A^{\prime}} \\
\xi_{A}
\end{array}\right)
$$

Denoting

$$
\gamma^{\alpha}=\sqrt{2}\left(\begin{array}{cc}
0 & \sigma^{\alpha} A^{\prime} \\
\bar{\sigma}^{\alpha A^{\prime} A} & 0
\end{array}\right)
$$

one can write the equation $D \psi= \pm m \psi$ as

$$
\left(p_{\alpha} \gamma^{\alpha} \mp m\right) \psi=0 \text {. }
$$

One can show, that the matrices $\gamma^{\alpha}$ satisfy the relation

$$
\gamma^{\alpha} \gamma^{\beta}+\gamma^{\beta} \gamma^{\alpha}=2 g^{\alpha \beta}
$$


writing

$$
\begin{aligned}
& \left(\begin{array}{cc}
0 & \sigma_{A A^{\prime}}^{\alpha} \\
\bar{\sigma}^{\alpha A^{\prime} A} & 0
\end{array}\right)\left(\begin{array}{cc}
0 & \sigma_{A A^{\prime}}^{\beta} \\
\bar{\sigma}^{\beta A^{\prime} A} & 0
\end{array}\right)+\left(\begin{array}{cc}
0 & \sigma^{\beta}{ }_{A A^{\prime}} \\
\bar{\sigma}^{\beta A^{\prime} A} & 0
\end{array}\right)\left(\begin{array}{cc}
0 & \sigma^{\alpha}{ }_{A A^{\prime}} \\
\bar{\sigma}^{\alpha A^{\prime} A} & 0
\end{array}\right)= \\
& \left(\begin{array}{cc}
\sigma_{A A^{\prime}}^{\alpha} \bar{\sigma}^{\beta A^{\prime} A}+\sigma_{A A^{\prime}}^{\beta} \bar{\sigma}^{\alpha A^{\prime} A} & 0 \\
0 & \bar{\sigma}^{\alpha A^{\prime} A} \sigma_{A A^{\prime}}^{\beta}+\bar{\sigma}^{\beta A^{\prime} A} \sigma_{A A^{\prime}}^{\alpha}
\end{array}\right)=2 g^{\alpha \beta}\left(\begin{array}{ll}
1 & 0 \\
0 & 1
\end{array}\right)
\end{aligned}
$$

using the formula $\sigma_{A A^{\prime}}^{\alpha} \bar{\sigma}^{\beta A^{\prime} A}+\sigma_{A A^{\prime}}^{\beta} \bar{\sigma}^{\alpha A^{\prime} A}=2 g^{\alpha \beta}$. The above relation are the anticommutation relations for the Dirac matrices. This way one obtains the Weyl representation of the Dirac spinors [11].

\section{REFERENCES}

[1] R. Abraham and J. E. Marsden. Foundations of Mechanics. The Benjamin/Cummings Publishing Company, second revised, enlarged and reset edition, 1978.

[2] H. Bacry. Lectures on Group Theory and Particle Theory. Gordon and Breach Science Publishers, 1977.

[3] A. O. Barut and R. Rączka. Theory of Group Representations and Applications. PWN Polish Scientific Publishers, Warszawa, second revised edition, 1980.

[4] R. J. Blattner. Quantization and representation theory. In Proc. Sympos. Pure Math., volume 26, pages 145-165. Amer. Math. Soc., R.I., 1974.

[5] P. Dirac. The quantum theory of the electron. Proc. Roy. Soc., 117:610-624, 1928.

[6] C. Duval, J. Elhadad, M. J. Gotay, J. Śniatycki, and G. M. Tuynman. Quantization and bosonic BRST theory. Annals of Physics, 206(1):1-26, 1991

[7] B. Kostant. Quantization and uintary representations. Lecture notes in mathematics, 170, 1970.

[8] G. W. Mackey. Induced Representaitons of Groups and Quantum Mechanics. Bejamin, 1968.

[9] R. Penrose and W. Rindler. Spinors and Space-Time, volume 1. Cambridge University Press, 1987.

[10] P. Renouard. Variétés Symplecitques et Quantification. PhD thesis, Université de Paris, 1969.

[11] S. S. Schweber. An Introduction to Relativistic Quantum Field Theory. Row, Peterson and Co., 1961.

[12] J. Śniatycki. Geometric Quantization and Quantum Mechanics. Springer-Verlag, 1980.

[13] J.-M. Souriau. Structure Des Systémes Dynamiques. Dunod, Paris, 1970.

[14] N. M. J. Woodhouse. Geometric Quantization. Clarendon Press, Oxford, second edition, 1992.

Department of Mathematics and Statistics, University of Calgary, 2500 UniverSity Dr. NW, Calgary AB T2N 1N4, Canada

E-mail address: bona@math.ucalgary.ca 